\title{
An interface between the POWHEG BOX and MADGRAPH5_AMC@NLO
}

\author{
Paolo Nason $^{1,2, \mathrm{a}}$, Carlo Oleari ${ }^{1,2, \mathrm{~b}}{ }_{(\mathbb{C})}$, Marco Rocco ${ }^{1,2, \mathrm{c}}$, Marco Zaro ${ }^{3, \mathrm{~d}}$ \\ ${ }^{1}$ Università di Milano-Bicocca, Piazza della Scienza 3, 20126 Milan, Italy \\ ${ }^{2}$ INFN, Sezione di Milano-Bicocca, Piazza della Scienza 3, 20126 Milan, Italy \\ ${ }^{3}$ INFN, Sezione di Milano and TIFLab, Via Celoria 16, 20133 Milan, Italy
}

Received: 28 August 2020 / Accepted: 15 October 2020 / Published online: 24 October 2020

(C) The Author(s) 2020

\begin{abstract}
In this paper we present a framework for developing POWHEG BoX generators using MADGRAPH5_AMC@NLO for the computation of the matrix elements. Within this framework, all the flexibility of MADGRAPH5_AMC@NLO for the generation of matrix elements for Standard Model processes and for several of its extensions can be exploited, as well as all features of the POWHEG Box framework, including the possibility of multijet merging without a merging scale (using the so called MiNLO approach). As a proof of concept, we develop a generator for the production of a spin- 0 Higgs-like boson in association with up to two jets, with CP-violating couplings.
\end{abstract}

\section{Contents}

1 Introduction . . . . . . . . . . . 1

2 Interface to MG5_AMC . . . . . . . . . . . . 2

2.1 Technical details . . . . . . . . . . . . 3

2.2 Distribution of the code . . . . . . . . . . . 4

3 A case study: $X_{0} j j$ production with CP-violating couplings ................ 4

3.1 Theoretical setup . . . . . . . . . . . . . 4

3.2 Generation of the code . . . . . . . . . . . 4

3.3 Simulation parameters . . . . . . . . . . 5

3.4 Phenomenology . . . . . . . . . . . . . 5

3.5 Reweighting . . . . . . . . . . . . . . . 8

3.6 MiNLO . . . . . . . . . . . . . . . . 10

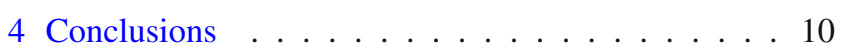

References.................. 11

a e-mail: paolo.nason@mib.infn.it

be-mail: carlo.oleari@mib.infn.it (corresponding author)

c e-mail: m.rocco10@campus.unimib.it

d e-mail: marco.zaro@mi.infn.it

\section{Introduction}

Next-to-leading-order (NLO) calculations for Standard Model (SM) and, sometimes, beyond-the-SM (BSM) processes, interfaced to parton shower (PS) generators, generally dubbed NLO+PS generators, are by now the methods of choice for the generation of event samples for signal and background processes at the LHC. This state of the art has been made possible, on the one side, by the formulations of general methods for computing NLO corrections [1,2], and, on the other, by the theoretical development of algorithms for interfacing fixed order calculations with parton shower generators [3-8]. These algorithms were implemented in software packages for the automatic computation of NLO corrections [9-13], and for the automatic implementation of NLO+PS generators $[9,14-17]$ that considerably ease the construction of generators for new processes.

MADGRAPH5_AMC@NLO, often abbreviated to MG5_AMC in the following, is a framework where automation has been pushed to the highest level. In fact, a user without any knowledge of NLO calculations or NLO+PS implementations can easily generate samples of parton-level events with NLO+PS accuracy, within the MC@NLO procedure. These events can be then directly fed into a PS generator, such as PYTHIA or HERWIG. The MG5_AMC framework is not restricted to the case of SM processes. In fact, it is possible to employ any user-defined model if this is provided in the socalled UFO format [18], for example as generated by FEYNRULES $[19,20]$. In particular, in order to undertake an NLO computation, the model should include the relevant UV and rational counterterms (both needed for the numerical evaluation of the one-loop matrix elements), which can be also automatically computed with FeYNRULES+NLOCT [21]. Furthermore, the FEYNRULES+MG5_AMC framework has been recently extended in order to fully support the supersymmetric case, including the implementation of different renormal- 
isation conditions [22], and the use of the so-called diagram removal and diagram subtraction techniques when intermediate resonances are present. NLO capabilities for BSM processes have been proven successful for a number of processes, see Ref. [22] and references therein.

The POWHEG method allows to generate events with positive weights and, because of this, it has become the method of choice when large samples of events are needed. In fact, in view of the large amount of computer resources needed for detector simulation, the experimental collaborations cannot afford to use the larger samples that are required when negative weights are present. ${ }^{1}$ The method has been also extended with the introduction of some theoretical developments of general interest. One of them deals with the generation of multijet samples that maintain a certain level of accuracy, even when some of the jets become unresolved $[24,25]$. This approach has also led to the development of NNLO+PS generators, i.e. generators where nextto-next-to-leading-order (NNLO) calculations are interfaced to parton showers [26-28]. Another development has been the extension of the POWHEG method for the inclusion of processes with decaying coloured resonances, which is capable of handling the interference of the emitted radiation generated in production and decay [32]. ${ }^{3}$

The POwHEG Box framework automatises the construction of NLO+PS generators, once the matrix elements are available. In the early POWHEG Box processes, the matrix elements were obtained from the authors of specific calculations. A considerable leap in the construction of the matrix elements took place when an interface of the POWHEG Box to MADGRAPH4 was set up [34], allowing for the implementation of all tree-level ingredients required by a given NLO process. After this development, the only missing ingredient for an NLO calculation in the POWHEG BOX was the virtual contribution. Later, interfaces to automatic generators of virtual processes were also developed in Refs. [35,36] for GosAM, and in Ref. [37] for OPENLOOPS.

As of now, an interface to the matrix-element generator that is available within the MG5_AMC package has not been developed. The main obstacle is the fact that MG5_AMC is built as a single package that aims at the production of partonic events, at difference with MADGRAPH4, that was initially conceived for the generation of tree-level matrix elements. An interface between the matrix-element generator of MG5_AMC and the POWHEG BoX is also highly desirable since many BSM processes are available within MG5_AMC.

\footnotetext{
${ }^{1}$ A variant of the MC@NLO method for drastically reducing the negative weight fraction has appeared in Ref. [23].

${ }^{2}$ Alternative methods for multijet merging have been presented in Refs. $[7,8,29]$. Alternative methods for NNLO+PS accuracy have been proposed in Refs. [30,31].

${ }^{3}$ See also Ref. [33].
}

In order to exploit the full capabilities of the MG5_AMC package, such interface should also build, in addition to the virtual contribution, all the necessary tree-level matrix elements: the Born, the colour- and spin-correlated Born, and the real matrix elements.

The purpose of the present work is to present an interface between the MG5_AMC matrix-element generator and the POWHEG BOX. The structure of the interface is such that developments in MG5_AMC and POWHEG can remain independent to a large extent. For this reason, our aim is not to construct a framework that is automatised at the same level as the full MG5_AMC package itself, but rather to build an MG5_AMC extension that makes the NLO matrix elements readily available to POWHEG. Thus, progresses on the POWHEG Box side and on the MG5_AMC side can take place independently, which is a considerable advantage in view of the way in which theoretical projects are developed. Furthermore, this kind of interface allows generalisations to other NLO+PS frameworks, that may also benefit from it for the implementation of the matrix elements.

The paper is organised as follows. In Sect. 2 we describe the interface and we give some technical details on how to use it and how to distribute the generated code. In Sect. 3 we consider, as a case study, the production of a spin- 0 boson $X_{0}$ plus two jets. In particular, we present a few distributions able to characterise the $X_{0}$ boson CP properties and we discuss some features connected to the POWHEG Box reweighting feature. We also show a few distributions obtained with the MiNLO approach. Finally, in Sect. 4 we draw our conclusions.

\section{Interface to MG5_AMC}

The new interface between POwHEG and MG5_AMC uses the capability of the latter to provide tree-level and oneloop matrix elements to be used by the former. The interface itself is a plugin for MG5_AMC: as such, it does not require any modification of the core code and it works with any recent version of MG5_AMC. ${ }^{4}$ It re-organises the output of MG5_AMC in a format which is suitable for the POWHEG Box [14], closely following what is described in Ref. [34]. At variance with what is discussed there, no external providers for the one-loop matrix elements are needed. Rather, one-loop matrix elements are directly generated by MG5_AMC thanks to the MADLOOP module [9,39], which encapsulates several different strategies, such as integrand reduction [40], Laurent-series expansion [41] and tensor-

\footnotetext{
${ }^{4}$ Versions 2.6 and onward are fully supported, for what concerns QCD corrections. The extension of the interface to more recent releases able to deal with electro-weak corrections (from version 3) [38] is left for future work.
} 
integral reduction [42-44], as implemented in different computer libraries [45-48] and improved by an in-house implementation of the OPENLOOPS method [10]. Thus, by fully exploiting the capabilities of MADLOOP, the evaluation of virtual matrix elements and the assessment of the numerical stability of the results are granted. Along with the matrix elements, the relevant helicity routines are also provided, in the ALOHA format [49].

\subsection{Technical details}

The interface plugin, dubbed MG5AMC- PWG, is publicly available. ${ }^{5}$ Its usage is very simple, as one only needs to copy (or link) the MG5 aMC_PWG folder inside the PLUGIN directory of MG5_AMC. Please refer to the README file enclosed in the package for conditions of usage and instructions.

The plugin can be loaded by launching, within the MG5_AMC installation directory,

./bin/mg5_aMC --mode=MG5aMC_PWG

in a command shell. In order to generate the code for a specific process at NLO QCD accuracy, the usual syntax of MG5_AMC should be employed. For example, in the case of top-pair production, the syntax is the following:

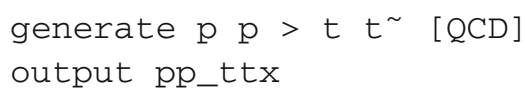

where pp_tt $x$ is the name (chosen by the user) of the directory where the code will be created. During the execution of the generate command, the MG5AMC- PWG plugin checks whether an installation of the POWHEG BOX V2 is available on the system and asks for its installation path (this is needed only once).

When this stage is concluded, the user can quit MG5_AMC and finds the MG5_AMC code for the Born, real and virtual contributions in the pp_ttx directory, in addition to a few basic PowHEG Box V2 files. In particular, the Born.f, real.f and virtual. $f$ files are ready to be used. Also the init_processes.f file can be used as it is, but can be also modified if particular features of the POWHEG BOX V2 need to be activated and initialised.

A few comments about the other files are in order:

- The Born_phsp. $\mathrm{f}$ file is just a place holder. It needs to be replaced by the actual phase-space generator for the process at hand. Examples of Born_phsp. $\mathrm{f}$ implementations can be found in the processes already imple-

\footnotetext{
5 Details can be found at https://code.launchpad.net/ $\sim$ mg5amc-pwg-team/mg5amc-pwg/v0, while the code can be downloaded with the command: bzr branch lp: mg5amc-pwg-team/mg5amc-pwg/v0. The installation of the revision control system bazaar is required.
}

mented in the PowHEG Box V2. In the current setup, a subroutine born_suppression should be also implemented in the Born_phsp. $\mathrm{f}$ file. This function is used at the integration stage to suppress divergences when present at the Born level, i.e. when there are jets and photons.

- The call of the setpara ("param_card.dat") routine in the init_couplings.f file initialises the parameters listed in the Cards/param_card.dat file to the corresponding values, according to the UFO model [18] used in MG5_AMC. ${ }^{6}$ It is also possible to assign a value to a MG5_AMC parameter at execution time. An example of this can be found in the init_couplings.f file for the process $X_{0} j j$, that we discuss in Sect. 3. In this file we reassign the value of $\cos \alpha$, the CP-mixing parameter that appears in the Lagrangian of Eq. (3.3). This parameter is indicated with cosa in the Cards/param_card.dat file, and is initialised to the value specified in this file, if no further action is taken. In order to reassign its value at execution time, we change the values of the internal MG5_AMC variables, mdl_cosa and mp_mdl_cosa (for double and quadruple precision), that encode this parameter.

After any reassignment of the MG5_AMC parameters, the user has to call the coup routine in order to recompute all the dependent variables.

- In order to have full consistency between the MG5_AMC amplitudes and what is computed by the POWHEG BOX V2, all the physical parameters used by the POWHEG BoX V2 should be set starting from those assigned or computed by MG5_AMC. An example of this is the list of the external-particle masses, kn_masses, used by POWHEG BOX V2 when generating the kinematics of the event. Using $t \bar{t}$ production as example, kn_masses should be set to

$\backslash\left(0,0, \mathrm{mdl} \_m t, \mathrm{mdl} \_\mathrm{mt}, 0 \backslash\right)$

in init_couplings.f or Born_phsp.f, where mal_mt is the mass of the top quark used inside MG5_AMC, the first two entries are the masses of the incoming particles, and the last massless particle is the radiated one, when computing the real contribution.

- The interface also builds a script file, prepare_run_ dir, that is useful to create a directory where the produced code can be executed. For example, by typing the command

\footnotetext{
${ }^{6}$ It should be noted that the Cards/param_card. dat file is not read at execution time. Rather, it is parsed at compilation time into a FORTRAN include file, which is then compiled together with the code. Hence, after any parameter modification within this file, the main executable has to be recompiled.
} 
. /prepare_run_dir test

a directory test is created. This directory contains all the relevant links to the MG5_AMC code and a template of the powheg.input file, required by the PowHEG Box V2. This last file should then be changed and modified according to the process at hand.

The POWHEG process generated along these lines can be completed with all sorts of features that are commonly used in the POWHEG Box V2. For example, one can activate the MiNLO option for processes with associated jets, or use the damping option to separate the real contributions into two parts, along the lines of what was suggested in the original POWHEG paper [4], and applied for the first time in Ref. [50].

\subsection{Distribution of the code}

A process generated with this interface to MG5_AMC cannot be distributed as a usual POWHEG Box process, since the searching path of the linked libraries are written in several files at generation time.

An author can distribute the instructions for MG5_AMC, needed in order to generate the process, and the actual files, that overwrite the place holders created by the interface plugin. In this way, all relevant paths point to the right directories in the user computer.

Alternatively, the author of the process may provide a script file that automatically executes all these tasks, helping the installation phase.

\section{A case study: $X_{0} j j$ production with CP-violating couplings}

For our case study, we considered the production of a spin0 boson $X_{0}$ (a Higgs-like boson) that couples to a massive top quark, produced via gluon fusion, and accompanied by two jets, in the heavy-top-mass limit. We discuss a few distributions able to characterise the $X_{0}$ boson CP properties, and discuss a few results obtained using the POWHEG BOX $\mathrm{V} 2$ reweighting feature. We also present a few distributions obtained with the MiNLO method.

\subsection{Theoretical setup}

The theoretical framework of this study is fully inherited from what was done in Ref. [51], where the process was studied at NLO in QCD. In particular, in the heavy-top-mass limit, the $\mathrm{CP}$ structure of the $X_{0}$-top interaction characterises the effective $g g X_{0}$ vertex. The starting point is the effective Lagrangian
$\mathcal{L}_{0}^{t}=-\bar{\psi}_{t}\left(k_{H t t} g_{H t t} \cos \alpha+i k_{A t t} g_{A t t} \sin \alpha \gamma_{5}\right) \psi_{t} X_{0}$,

where $X_{0}$ is the spin- 0 boson, $\psi_{t}$ the top-quark spinor, $\alpha$ the CP-mixing angle parameter $(0 \leq \alpha \leq \pi), k_{H t t}$ and $k_{A t t}$ the real coupling parameters and

$g_{H t t}=g_{A t t}=\frac{m_{t}}{v}=\frac{y_{t}}{\sqrt{2}}$

the Yukawa couplings, with $v$ the vacuum expectation value.

The CP-even case, that will be labeled $0^{+}$, corresponds to the assignment $\cos \alpha=1$, namely to the SM scenario, while the CP-odd case, labeled $0^{-}$, to $\cos \alpha=0$. A CP-mixed case, $0^{ \pm}$, where the spin- 0 boson receives contributions from both a scalar and a pseudoscalar state, is also taken into account by setting $\cos \alpha=1 / \sqrt{2}$.

For our purposes, it will suffice to notice that the Higgs interaction with the gluons originates as an effective coupling induced by a top-quark loop. The relevant effective Lagrangian, in the Higgs Characterisation framework [52], reads

$$
\begin{aligned}
\mathcal{L}_{0, g}^{\text {loop }}= & -\frac{1}{4}\left(k_{H g g} g_{H g g} \cos \alpha G_{\mu \nu}^{a} G^{a, \mu \nu}\right. \\
& \left.+k_{A g g} g_{A g g} \sin \alpha \epsilon^{\mu \nu \rho \sigma} G_{\mu \nu}^{a} G_{\rho \sigma}^{a}\right) X_{0},
\end{aligned}
$$

where $G_{\mu \nu}^{a}$ is the gluon field strength and

$k_{H g g}=-\frac{\alpha_{\mathrm{S}}}{3 \pi v}, \quad k_{A g g}=\frac{\alpha_{\mathrm{S}}}{2 \pi v}$.

The theoretical setup is made available online in the FEYNRULES [20] repository as a UFO model named HC_NLO_XO [51,53-55], which is in fact the one used for our case study.

\subsection{Generation of the code}

In order to generate the code, we have first to install the UFO model HC_NLO_XO_UFO.zip under the models directory of the MG5_AMC version being used. We have then followed the procedure described in Sect. 2.1 for the generation of the code, and given the following commands to MG5_AMC:

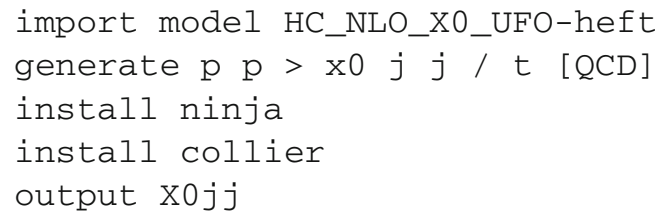

where we have also inserted the command lines to install NINJA [46] and COLLIER [48], that are optional and need to be installed just once.

We have then overwritten the Born_phsp. f file generated by the interface with the Born_phsp. f from the $H j j$ 
POWHEG Box V2 process, taking care of assigning to the PowHEG variables hmass and hwidth (the mass and width of the Higgs-like boson) the MG5_AMC values, mal_mx0 and $\mathrm{mal} \_w \times 0$ respectively.

In order to ease the installation procedure, we provide a tarball file that needs to be inflated in the installation directory. This file contains all the modified files that replace the place holders.

\subsection{Simulation parameters}

We have performed a simulation for the LHC, running at a centre-of-mass energy of $\sqrt{S}=13 \mathrm{TeV}$. The mass of the spin-0 boson $X_{0}$ has been set equal to $125 \mathrm{GeV}$. We have chosen the NNPDF2.3 (NLO) set [56] for the parton distribution functions, within the LHAPDF interface [57,58].

The differential cross section for $X_{0} j j$ production is already divergent at the Born level, unless a minimum set of generation cuts is imposed on the transverse momentum of the final-state jets and on their invariant mass. Alternatively, the divergences can be avoided if the code is executed with the MiNLO option activated. We have generated the kinematics of the underlying Born configurations imposing the following minimum set of cuts

$$
p_{\mathrm{T}}^{j_{k}}>10 \mathrm{GeV}, \quad k=1,2, \quad m_{j_{1} j_{2}}>10 \mathrm{GeV} .
$$

In the phenomenological study we perform in Sect. 3.4, we apply more stringent cuts, and we have checked that the results we present are insensitive to the generation cuts.

In order to integrate the divergent underlying Born cross section, the POWHEG BOX V2 can further apply a suppression factor at the integrand level. We stress that the final kinematic distributions are independent of this factor. ${ }^{7}$

\subsection{Phenomenology}

In this section we present results produced by the POWHEG Box V2 at the Les Houches Event (LHE) level, i.e. after the emission of the first radiation, accurate at NLO for large transverse momentum, and with leading-logarithmic accuracy at small $p_{\mathrm{T}}$, due to the presence of the POWHEG Sudakov form factor. The results are computed on samples of $3.2 \mathrm{M}$ events.

The renormalisation and factorisation scales are set to

$\mu_{\mathrm{R}}=\mu_{\mathrm{F}}=\frac{H_{\mathrm{T}}}{2}$

where $H_{\mathrm{T}}$ is the sum of the transverse masses of the particles in the final state.

\footnotetext{
7 We have set bornsupp fact to $30 \mathrm{GeV}$ in our simulation.
}

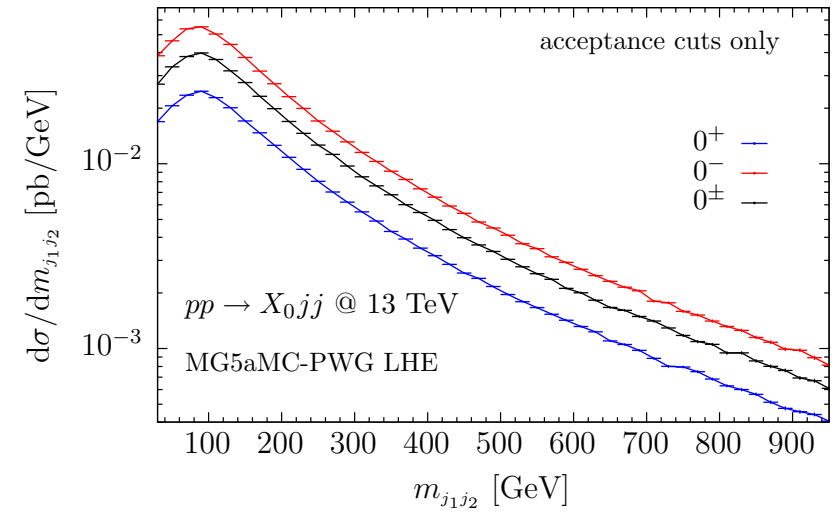

Fig. 1 Differential cross section as a function of the invariant-mass distribution of the two leading jets in $p p \rightarrow X_{0} j j$ for the three CP scenarios. The blue curve corresponds to the CP-even scenario with $\cos \alpha=1$, the red curve to the CP-odd scenario with $\cos \alpha=0$ and the black curve to the mixture of the $0^{+}$and $0^{-}$scenarios with $\cos \alpha=$ $1 / \sqrt{2}$

Jets are reconstructed employing the anti- $k_{\mathrm{T}}$ algorithm [59] via the FASTJET implementation [60], with distance parameter $R=0.4$, and the two leading jets are required to have transverse momentum and pseudorapidity such that

$p_{\mathrm{T}}^{j_{k}}>30 \mathrm{GeV}, \quad\left|\eta_{j_{k}}\right|<4.5, \quad k=1,2$.

Events that do not pass this minimum set of acceptance cuts are discarded.

In Fig. 1 we plot the differential cross section for $X_{0} j j$ production as a function of the invariant mass of the two leading jets, $m_{j_{1} j_{2}}$, for three different CP scenarios: $\mathrm{CP}$ even $\left(0^{+}\right), \mathrm{CP}$ odd $\left(0^{-}\right)$and a mixture of the two $\left(0^{ \pm}\right)$. The shapes of the three spectra are very similar among each other. Since a cut on the invariant mass of the dijet system enhances the discriminating power among different $\mathrm{CP}$ scenarios [61], the fact that the three spectra have similar shapes implies that the cut acts in a similar way on each of them. Typically a cut on $m_{j_{1} j_{2}}$ enhances the contributions coming from the exchange of a gluon in the $t$ channel, and these contributions are more sensitive to the CP properties of the $X_{0}$ boson.

In the following plots we impose an additional cut on the dijet mass. In particular, we consider the two cases where

$m_{j_{1} j_{2}}>250 \mathrm{GeV}$ and $m_{j_{1} j_{2}}>500 \mathrm{GeV}$.

In addition, since we are interested in shape comparisons among different $\mathrm{CP}$ scenarios, we normalise each curve to one.

In Figs. 2 and 3 we plot the transverse momentum and pseudorapidity of the $X_{0}$ boson, and in Figs. 4 and 5 we show the transverse momentum and pseudorapidity of the leading jet. The increase of the cut on the dijet mass hardens the $p_{\mathrm{T}}$ spectrum of the $X_{0}$ boson and the leading jet $j_{1}$. Moreover, 

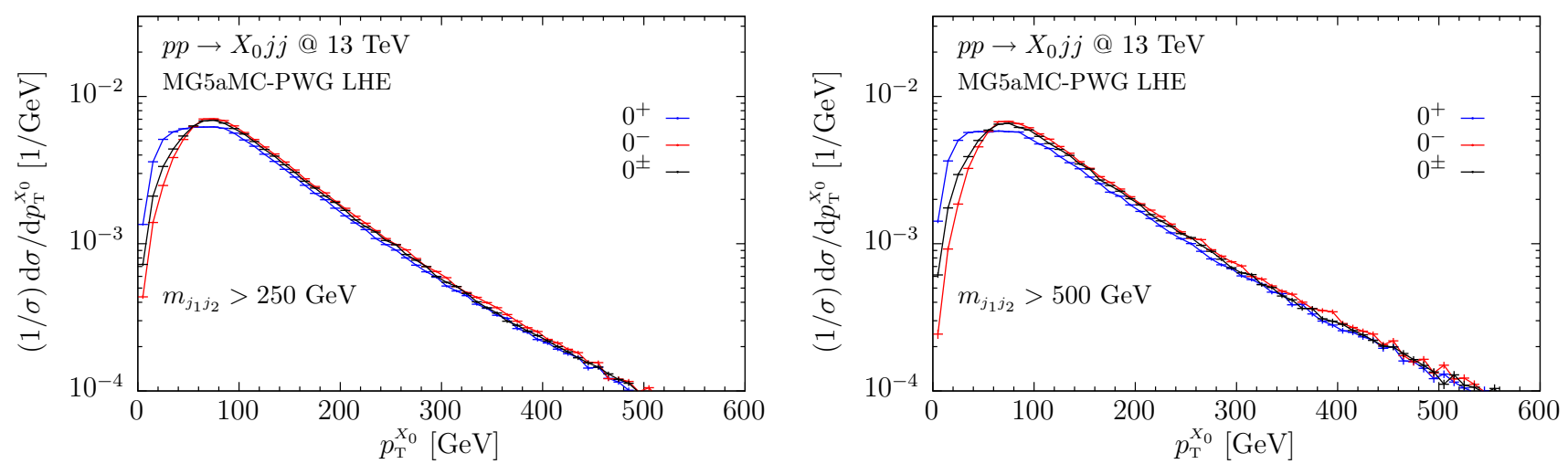

Fig. 2 Normalised differential cross section as a function of the transverse momentum of the spin- 0 boson $X_{0}$, for the three CP scenarios. On the left panel, a cut of $250 \mathrm{GeV}$ is imposed on the dijet mass, while on the right panel a cut of $500 \mathrm{GeV}$ is applied. The colour code is the same as in Fig. 1
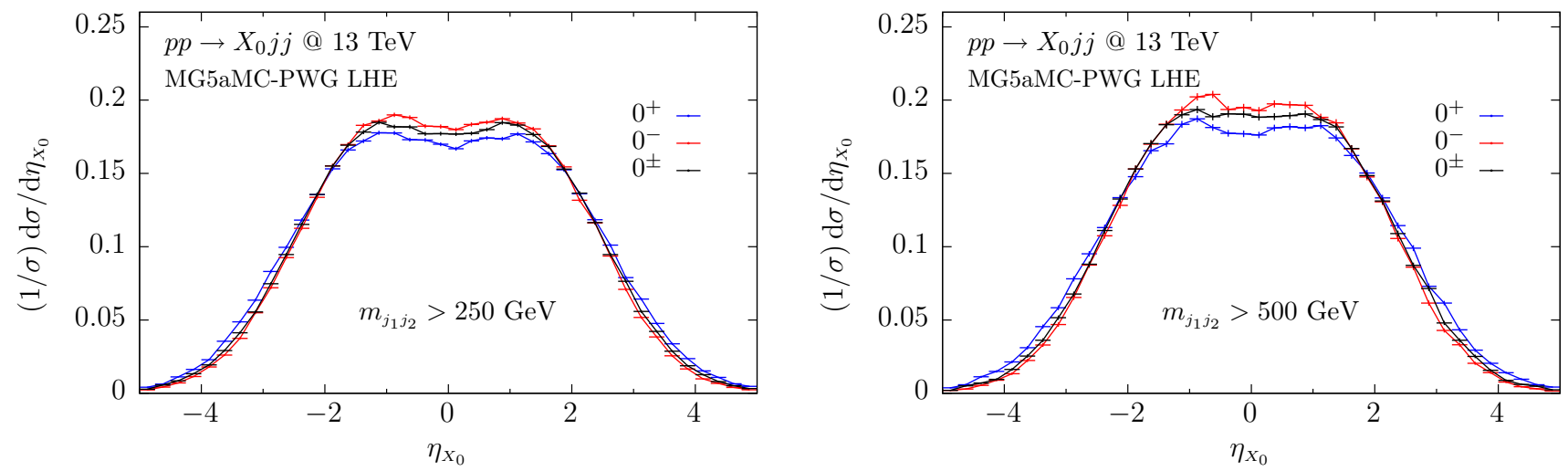

Fig. 3 Normalised differential cross section as a function of the pseudorapidity of the spin- 0 boson $X_{0}$, for the three CP scenarios. On the left panel, a cut of $250 \mathrm{GeV}$ is imposed on the dijet mass, while on the right panel a cut of $500 \mathrm{GeV}$ is applied. The colour code is the same as in Fig. 1
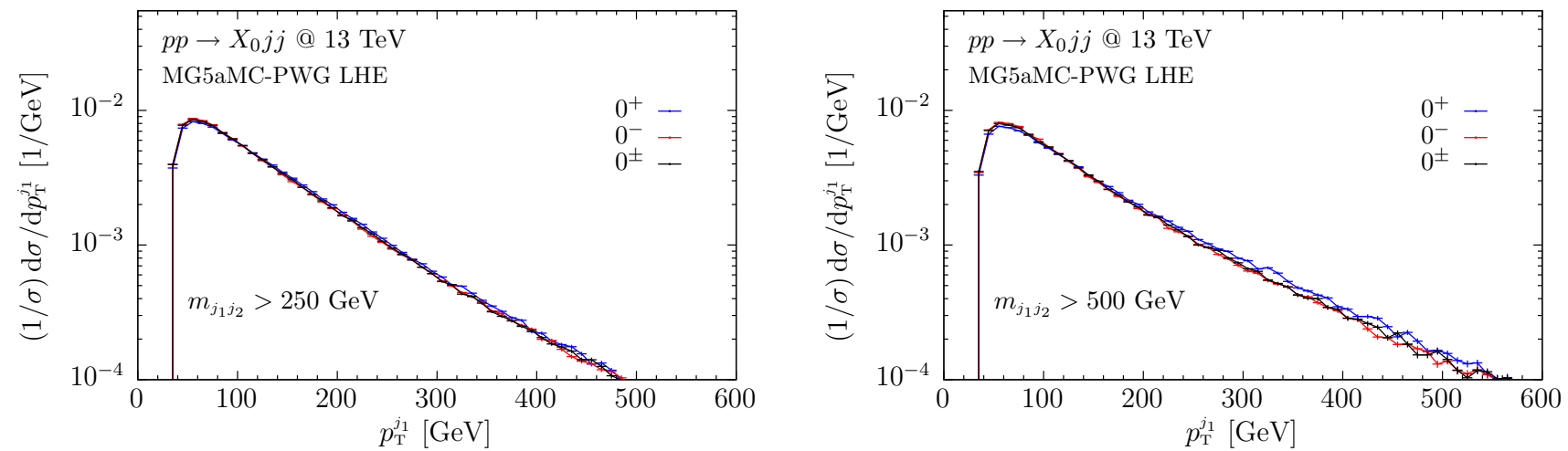

Fig. 4 Normalised differential cross section as a function of the transverse momentum of the leading jet, for the three CP scenarios. On the left panel, a cut of $250 \mathrm{GeV}$ is imposed on the dijet mass, while on the right panel a cut of $500 \mathrm{GeV}$ is applied. The colour code is the same as in Fig. 1

there are only mild differences among the three CP scenarios in the $X_{0}$ distributions at low transverse momentum and in the central pseudorapidity region, with a modest enhancement when the dijet-mass cut increases. No substantial differences are present in $p_{\mathrm{T}}^{j_{1}}$ and $\eta_{j_{1}}$, also in agreement with what is found in Ref. [51]. ${ }^{8}$

\footnotetext{
8 A possible concern is to what extent the effective-field-theory (EFT) Lagrangian of Eq. (3.3) produces sound results in the high-energy regimes, since it describes the full theory in the heavy-top-quark limit. From the exact calculation of Ref. [62], it is known that the EFT closely reproduces the $m_{j_{1} j_{2}}$ spectrum even in the very high invariant-mass
} 

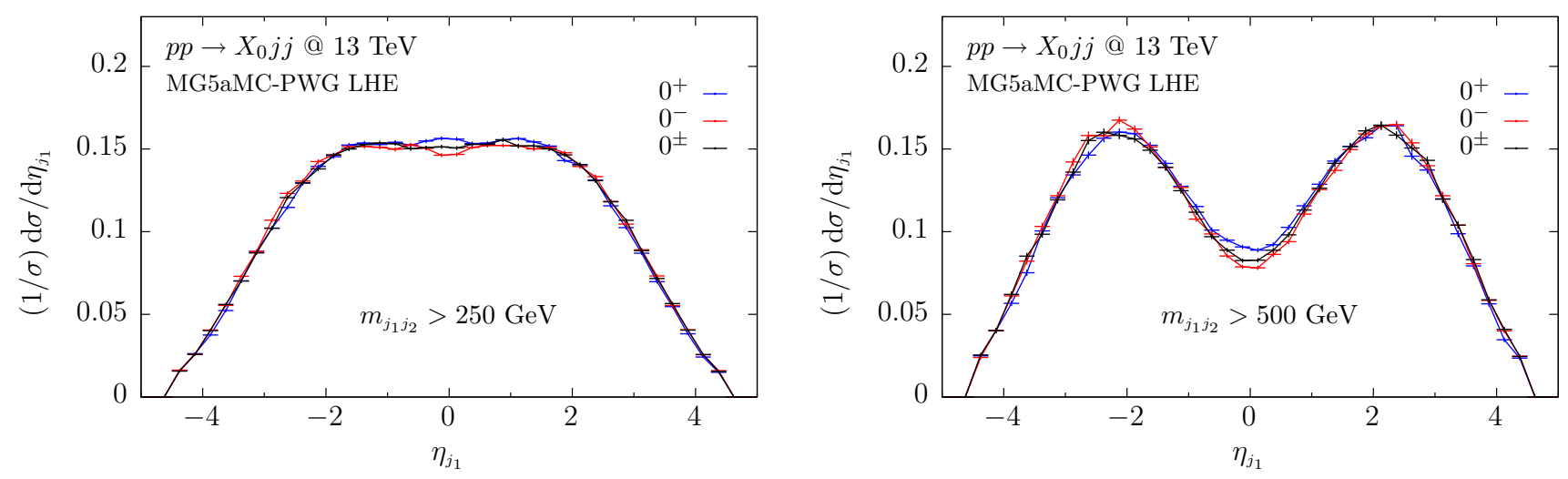

Fig. 5 Normalised differential cross section as a function of the pseudorapidity of the leading jet, for the three CP scenarios. On the left panel, a cut of $250 \mathrm{GeV}$ is imposed on the dijet mass, while on the right panel a cut of $500 \mathrm{GeV}$ is applied. The colour code is the same as in Fig. 1
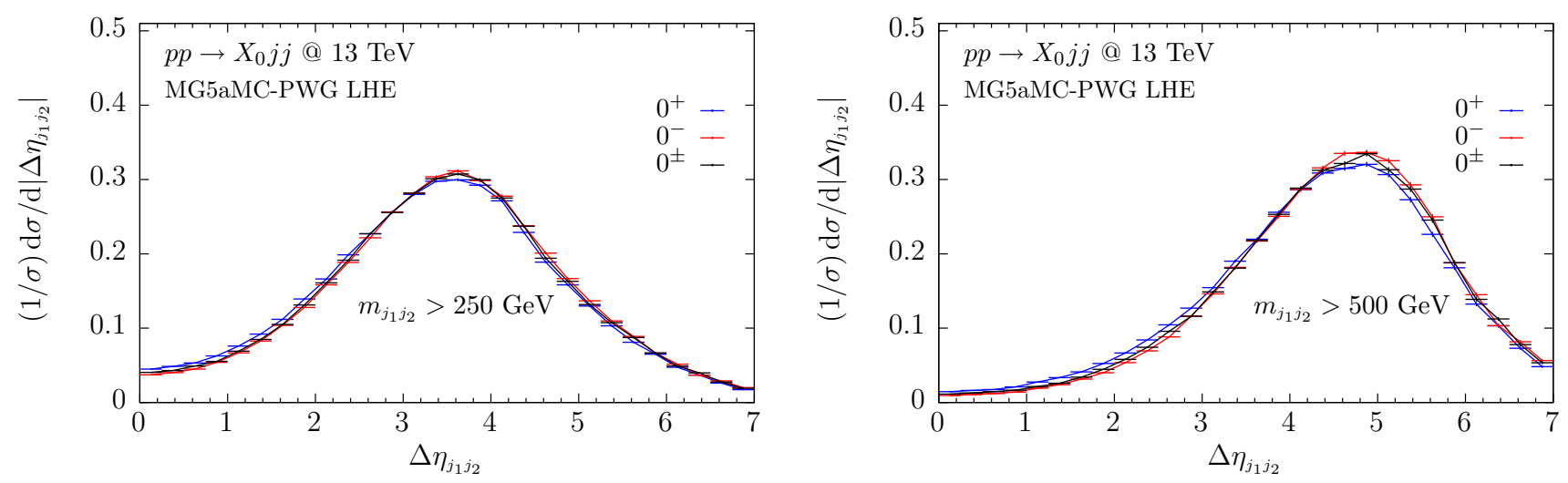

Fig. 6 Normalised differential cross section as a function of the pseudorapidity separation of the two leading jets (see Eq. (3.9)), for the three CP scenarios. On the left panel, a cut of $250 \mathrm{GeV}$ is imposed on the dijet mass, while on the right panel a cut of $500 \mathrm{GeV}$ is applied. The colour code is the same as in Fig. 1

The most sensitive observables to the CP coupling of the $X_{0}$ boson to the top quark in gluon fusion are dijet-correlation variables [61,64-70]. As displayed in Fig. 6, no significant differences are seen in the differential cross sections as a function of the pseudorapidity separation of the two leading jets

$\Delta \eta_{j_{1} j_{2}}=\left|\eta_{j_{1}}-\eta_{j_{2}}\right|$

Instead, when the differential cross sections are expressed as a function of the azimuthal-angle separation, the $\mathrm{CP}$ nature of the coupling is more evident [61]. In fact, the shape of the differential cross sections as a function of $\Delta \phi_{j_{1} j_{2}}$ are very different, as shown in Fig. 7, where we have defined (modulo $2 \pi$ )

region. However, the EFT approximation breaks down when the transverse momenta of the jets are larger than the top mass [63], overestimating the exact prediction when $p_{\mathrm{T}}^{j_{1}}$ is larger than the top mass. Since the region of interest for discriminating the $\mathrm{CP}$ properties is at low transverse momentum, we can trust the results obtained within the EFT approach.
$\Delta \phi_{j_{1} j_{2}}=\left|\phi_{j_{1}}-\phi_{j_{2}}\right|$

where the azimuth of a jet is computed as

$\phi_{j_{k}}=\arg \left(\mathbf{p}^{j_{k}} \cdot \hat{y}+i \mathbf{p}^{j_{k}} \cdot \hat{x}\right), \quad k=1,2$,

with $\mathbf{p}^{j_{k}}$ the tri-momentum of the jet $k$ and $\hat{x}(\hat{y})$ the unit vector along the $x(y)$-axis direction.

As pointed out in Refs. [65,71], a more CP-sensitive observable (especially for the maximal mixing scenario of $\cos \alpha=1 / \sqrt{2}$ considered here) is the oriented azimuthal separation of the two hardest jets. This variable contains information not only on the azimuthal separation of the two jets but also on the sign of the azimuthal angle. We have adopted the definition of this variable of Ref. [72], namely

$\Delta \phi_{j_{1} j_{2}}^{\text {or }} \equiv \frac{\left(\hat{\mathbf{p}}_{\mathrm{T}}^{j_{1}} \times \hat{\mathbf{p}}_{\mathrm{T}}^{j_{2}}\right) \cdot \hat{z}}{\left|\left(\hat{\mathbf{p}}_{\mathrm{T}}^{j_{1}} \times \hat{\mathbf{p}}_{\mathrm{T}}^{j_{2}}\right) \cdot \hat{z}\right|} \frac{\left(\mathbf{p}^{j_{1}}-\mathbf{p}^{j_{2}}\right) \cdot \hat{z}}{\left|\left(\mathbf{p}^{j_{1}}-\mathbf{p}^{j_{2}}\right) \cdot \hat{z}\right|} \arccos \left(\hat{\mathbf{p}}_{\mathrm{T}}^{j_{1}} \cdot \hat{\mathbf{p}}_{\mathrm{T}}^{j_{2}}\right)$, 

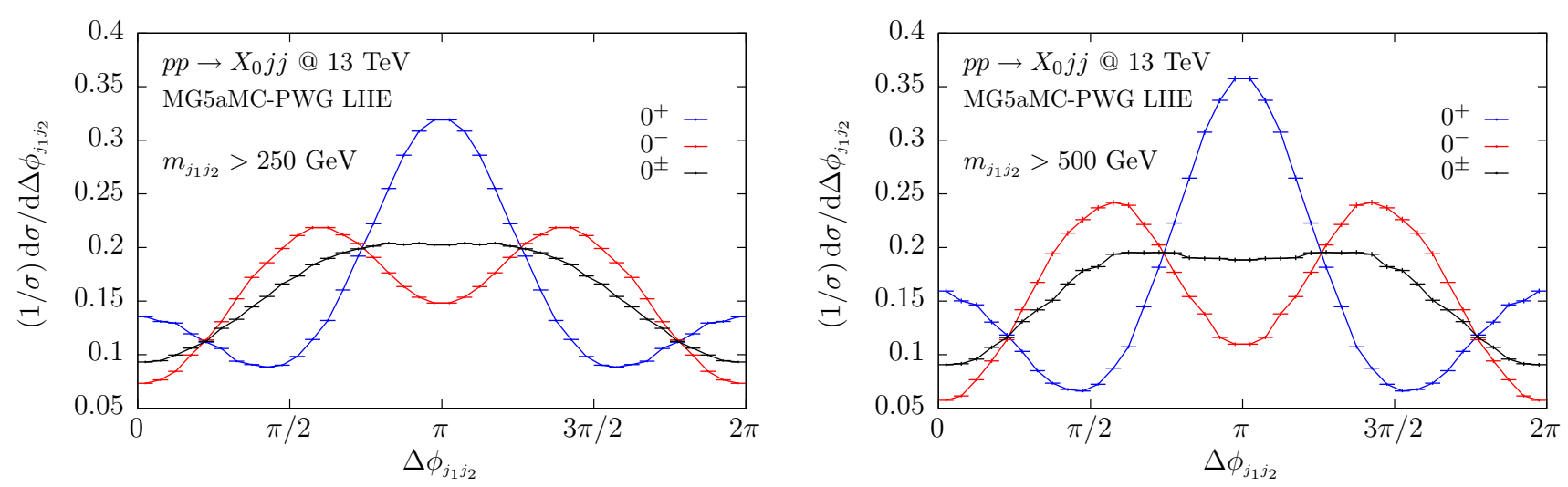

Fig. 7 Normalised differential cross section as a function of the azimuthal separation of the two leading jets (see Eq. (3.10)), for the three CP scenarios. On the left panel, a cut of $250 \mathrm{GeV}$ is imposed on the dijet mass, while on the right panel a cut of $500 \mathrm{GeV}$ is applied. The colour code is the same as in Fig. 1

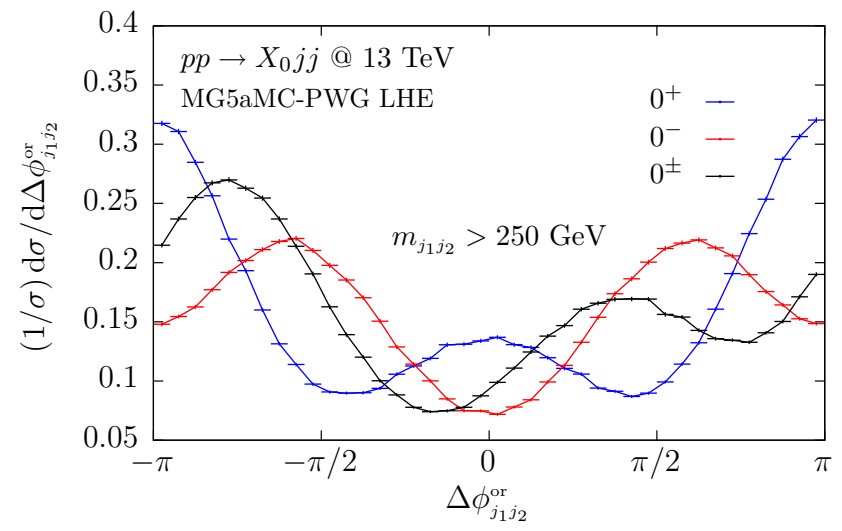

Fig. 8 Normalised differential cross section as a function of the oriented azimuthal separation of the two leading jets, defined in Eq. (3.12), for the three $\mathrm{CP}$ scenarios. On the left panel, a cut of $250 \mathrm{GeV}$ is imposed

where $\hat{\mathbf{p}}_{\mathrm{T}}^{j_{k}}$ is the jet transverse momentum, normalised to one, and $\hat{z}$ is the unit vector along the $z$-axis direction.

The differential cross sections for the three different $\mathrm{CP}$ scenarios considered in this paper, as a function of $\Delta \phi_{j_{1} j_{2}}^{\text {or }}$, are shown in Fig. 8, and their shapes are visibly different.

In particular, the oriented azimuthal separation can also distinguish between the two scenarios with $\cos \alpha=1 / \sqrt{2}$ and $\cos \alpha=-1 / \sqrt{2}$, as illustrated in Fig. 9, while $\Delta \phi_{j_{1} j_{2}}$ cannot distinguish between them.

\subsection{Reweighting}

In this section we present a few results obtained with the POWHEG BOX V2 reweighting feature. We have reweighted two of the event samples that we have produced: the scalar and the mixed one. We have then compared the reweighted distributions with the original ones, i.e. those computed from

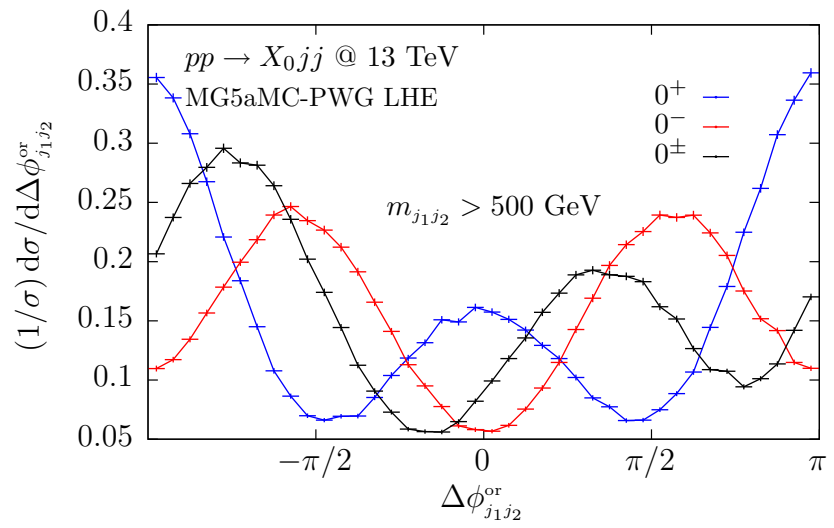

on the dijet mass, while on the right panel a cut of $500 \mathrm{GeV}$ is applied. The colour code is the same as in Fig. 1

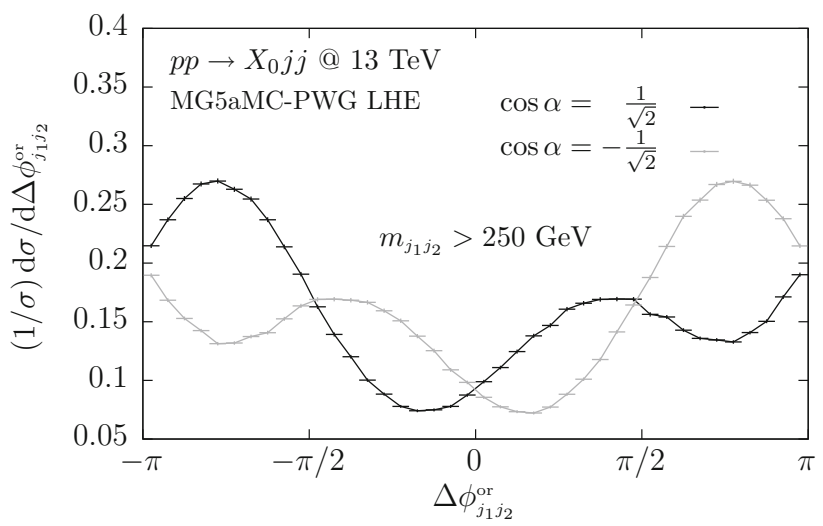

Fig. 9 Normalised differential cross section as a function of the oriented azimuthal separation of the two leading jets, defined in Eq. (3.12), for the two mixed CP scenarios with $\cos \alpha=1 / \sqrt{2}$ (black curve) and $\cos \alpha=-1 / \sqrt{2}$ (grey curve). A cut of $250 \mathrm{GeV}$ is imposed on the dijet mass 


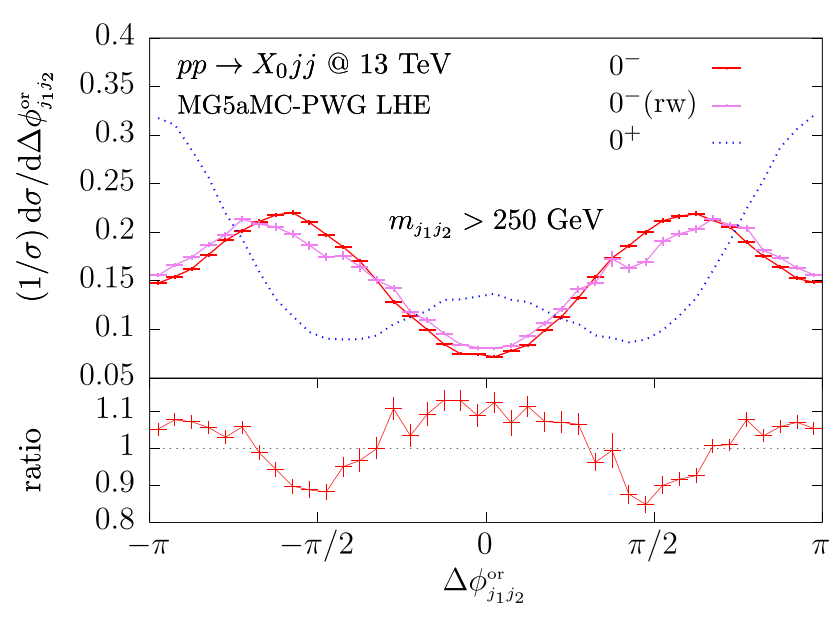

Fig. 10 Normalised differential cross section as a function of the oriented azimuthal separation of the two leading jets, defined in Eq. (3.12), with a cut of $250 \mathrm{GeV}$ imposed on the dijet mass. On the left panel, the pseudoscalar original distribution in red, the pseudoscalar as obtained by reweighting (rw) in pink, and the scalar one in dotted blue. On the

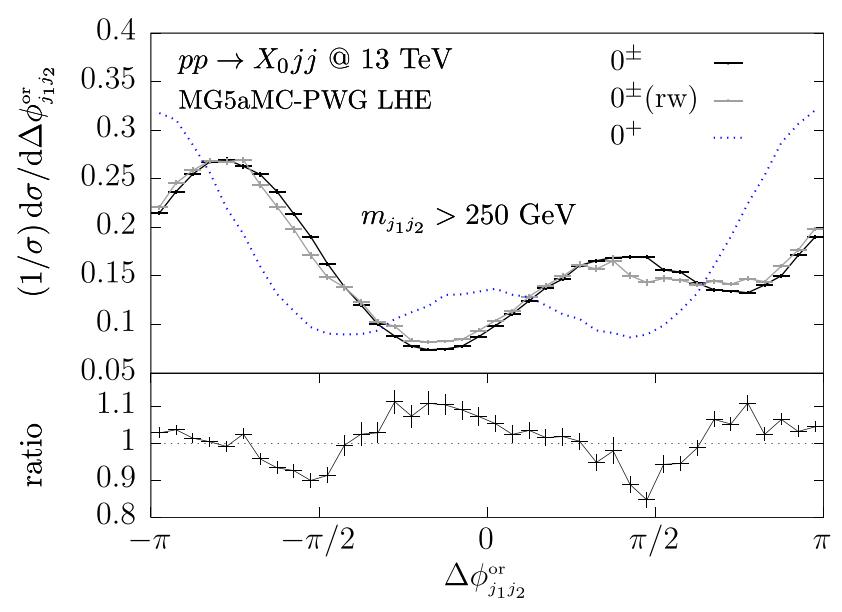

right panel, the $\mathrm{CP}$ mixed original distribution in black, the mixed as obtained by reweighting (rw) in gray, and the scalar one in dotted blue. The ratios between the distributions obtained by reweighting and the original ones are also shown
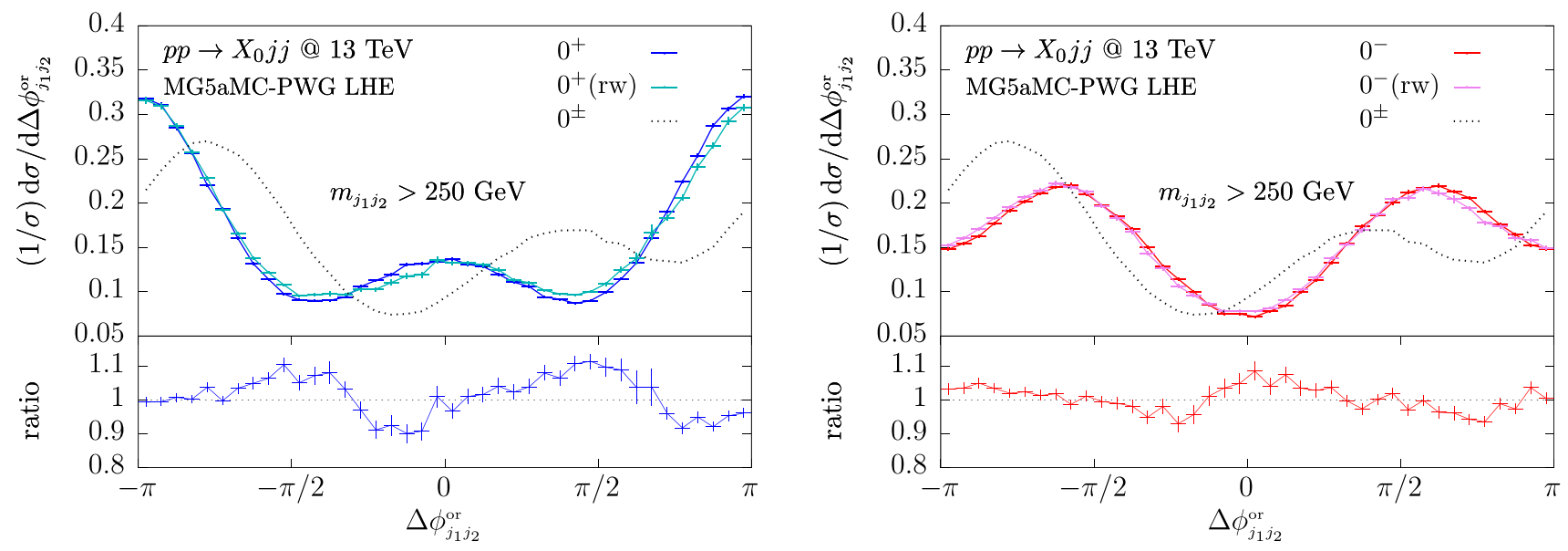

Fig. 11 Same as Fig. 10 but for the reweighting of the CP mixed sample to the scalar case (on the left) and to the pseudoscalar one (on the right)

the beginning with a given value of $\cos \alpha$. In particular, we have reweighted the scalar sample to the pseudoscalar and $\mathrm{CP}$ mixed cases, and we have reweighted the mixed sample to the scalar and pseudoscalar ones. We have found an overall good agreement between the reweighted and the original distributions, except for the distribution of the differential cross section expressed as a function of the oriented azimuthal angle, i.e. the distributions most sensitive to the value of the CP parameter $\cos \alpha$.

In Fig. 10 we compare three curves. The $\Delta \phi_{j_{1} j_{2}}^{\text {or }}$ distribution obtained from the original scalar sample is plotted in dotted blue, on both panels. This curve corresponds to the $0^{+}$ line on the left panel of Fig. 8. The scalar sample is reweighted to the pseudoscalar scenario on the left panel and to the mixed scenario on the right panel. The reweighted sample, indicated with "rw" in the figures, is then compared with the original distribution. The ratio of the last two curves is also plotted. In both cases, in correspondence to the minima of the $0^{+}$distribution, the discrepancy between the reweighted distribution and the original one is more than $-10 \%$, the minus sign to indicate that the distributions obtained by reweighting underestimate the original ones. The opposite is also true: when the $0^{+}$distribution has maxima that are not close to the maxima of the $0^{-}$and $0^{ \pm}$distributions, we have a discrepancy on the opposite side, up to $+10 \%$.

Similar conclusions can be drawn by reweighting the $0^{ \pm}$ sample, as illustrated in Fig. 11, in order to produce the differential cross section as a function of $\Delta \phi_{j_{1} j_{2}}^{\text {or }}$ for the $0^{+}$and $0^{-}$scenarios.

These differences can be explained by noticing that the minima of the above distributions are actually zeros at LO, and the production of events around these regions is then 


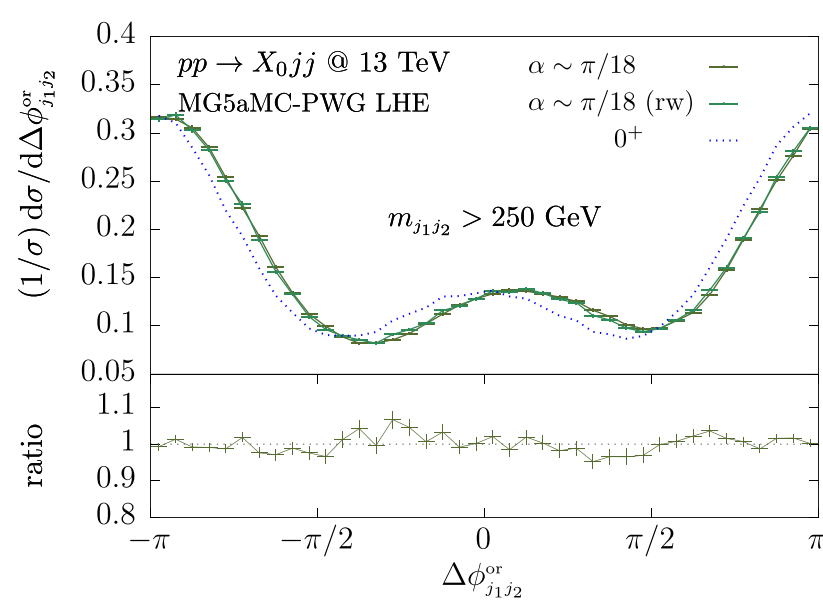

Fig. 12 Same as Fig. 10 but for the reweighting of the scalar sample to the CP scenario defined by $\cos \alpha=0.985$

suppressed. The reweighting procedure is not able to generate the correct distributions, if the starting one is very different from the final one, i.e., for example, going from $\alpha=0$ to $\alpha=$ $\pi / 2$, for the reweighting of the scalar case to the pseudoscalar one.

Otherwise, if the reweighting procedure is used to reweight distributions with similar values of the angle $\alpha$, the procedure correctly works. This is shown in Fig. 12, where the distribution computed with $\alpha=0$ is reweighted to the distribution with $\alpha \sim 10^{\circ} \sim \pi / 18$, and the agreement with the exact one is very good.

\subsection{MiNLO}

In this section we present a few results for the pseudoscalar $X_{0}$ production, obtained within the MiNLO procedure. Although all the cuts applied on the jets in the previous sections are completely removed, the differential cross sec-

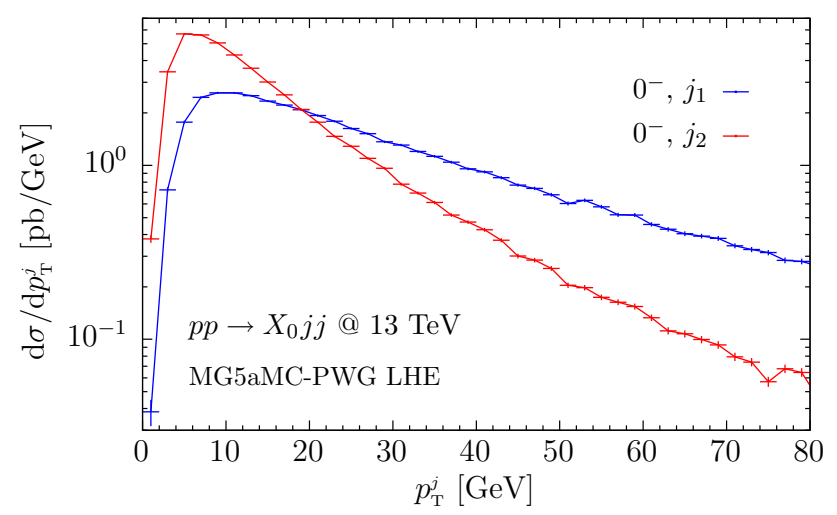

Fig. 13 On the left panel the inclusive differential cross section as a function of the transverse momentum of the hardest jet, in blue, and of the second-to-hardest one, in red. The CP scenario is defined by tions for inclusive quantities are finite, due to the presence of the MiNLO Sudakov form factor.

This is shown, for example, in Fig. 13, where we plot the inclusive differential cross section as a function of the transverse momentum of the hardest and of the second-tohardest jet, on the left panel, and the inclusive rapidity of the $X_{0}$ boson, on the right one.

Although finite, we cannot make any claim on the accuracy of these distributions, i.e. they do not reach the NLO accuracy of the MiNLO' method, described in Refs. $[25,73] .^{9}$

\section{Conclusions}

In this paper we have presented an interface between MADGRAPH5_AMC@NLO and the POWHEG Box V2, able to build a NLO + parton shower generator for Standard Model and many beyond-the-Standard-Model processes, in an automatic way.

The structure of the interface is such that future developments in MADGRAPH5_AMC@NLO and POWHEG BOX V2 remain independent to a large extent, so that it benefits from all the progresses coming from both sides. In fact, on the one side, MADGRAPH5_AMC@NLO provides the matrix elements for the Born, the colour- and spin-correlated Born, the real and the virtual contributions. On the other, the PowHEG Box uses these ingredients to generate events accurate at the $\mathrm{NLO}+$ parton shower level. In addition, the interface writes

\footnotetext{
${ }^{9}$ Our MG5aMC-PWG implementation is NLO accurate only for quantities involving two detected jets. The MiNLO procedure allows to obtain finite and LO accurate predictions for quantities with one or zero detected jets. Otherwise, the only available method able to reach NLO accuracy for one-jet inclusive distributions, for colour-singlet production with two associated jets within the PowHEG-MiNLO method, is the one illustrated in Ref. [73].
}

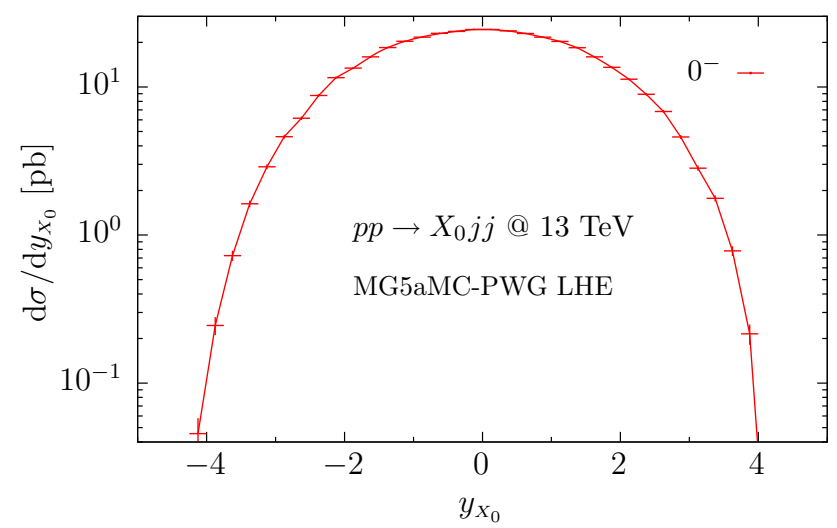

$\cos \alpha=0$, namely, the pseudoscalar case. On the right panel in red, the inclusive rapidity of the $X_{0}$ boson, for the same $\mathrm{CP}$ scenario as in the left panel. Both plots are obtained with MiNLO 
other files needed by the POWHEG Box V2. Some of them, as the list of processes, are fully finalised. Others, such as the phase-space generator, need to be adjusted in order to deal with the process at hand.

By now the interface only deals with processes for which we aim at NLO QCD accuracy. The extension including the electroweak corrections and the interface with the more recent version of the POWHEG BOX, i.e. the POWHEG BOX RES, is left as future work.

As a case study, using this interface we have generated the code for the production of a spin- 0 boson plus two jets, and we have computed a few kinematic distributions, sensitive to the CP properties of the coupling of the boson with a massive top quark. We have compared these distributions with known results in the literature and found full agreement. We have also presented a few results for the pseudoscalar case, obtained within the MiNLO approach.

Finally, we have tested the POwHEG Box reweighting feature. This procedure works fine for every kinematic distributions we have examined, but for the ones most sensitive to the CP nature of the $X_{0}$ boson. In fact, we have observed that it works if the reweighting is done from one distribution to another, with values of the mixing angle $\alpha$ not very different from each other.

Acknowledgements P.N. acknowledges support from Fondazione Cariplo and Regione Lombardia, Grant 2017-2070, and from INFN. We thank S. Frixione and F. Maltoni for useful discussions. We thank A. Gritsan for suggesting the process we implemented.

Data Availability Statement This manuscript has no associated data or the data will not be deposited. [Authors' comment: There are no associated data to our paper.]

Open Access This article is licensed under a Creative Commons Attribution 4.0 International License, which permits use, sharing, adaptation, distribution and reproduction in any medium or format, as long as you give appropriate credit to the original author(s) and the source, provide a link to the Creative Commons licence, and indicate if changes were made. The images or other third party material in this article are included in the article's Creative Commons licence, unless indicated otherwise in a credit line to the material. If material is not included in the article's Creative Commons licence and your intended use is not permitted by statutory regulation or exceeds the permitted use, you will need to obtain permission directly from the copyright holder. To view a copy of this licence, visit http://creativecomm ons.org/licenses/by/4.0/.

Funded by SCOAP ${ }^{3}$.

\section{References}

1. S. Frixione, Z. Kunszt, A. Signer, Three jet cross-sections to nextto-leading order. Nucl. Phys. B 467, 399 (1996). https://doi.org/10. 1016/0550-3213(96)00110-1. arXiv:hep-ph/9512328

2. S. Catani, M.H. Seymour, A general algorithm for calculating jet cross-sections in NLO QCD. Nucl. Phys. B 485, 291 (1997). https://doi.org/10.1016/S0550-3213(96)00589-5. https://doi.org/ 10.1016/S0550-3213(98)81022-5. arXiv:hep-ph/9605323
3. S. Frixione, B.R. Webber, Matching NLO QCD computations and parton shower simulations. JHEP 06, 029 (2002). https://doi.org/ 10.1088/1126-6708/2002/06/029. arXiv:hep-ph/0204244

4. P. Nason, A new method for combining NLO QCD with shower Monte Carlo algorithms. JHEP 11, 040 (2004). https://doi.org/10. 1088/1126-6708/2004/11/040. arXiv:hep-ph/0409146

5. S. Frixione, P. Nason, C. Oleari, Matching NLO QCD computations with Parton Shower simulations: the POWHEG method. JHEP 11, 070 (2007). https://doi.org/10.1088/1126-6708/2007/11/070. arXiv:0709.2092

6. S. Jadach, W. Placzek, S. Sapeta, A. Siódmok, M. Skrzypek, Matching NLO QCD with parton shower in Monte Carlo scheme-the KrkNLO method. JHEP 10, 052 (2015). https://doi.org/10.1007/ JHEP10(2015)052. arXiv:1503.06849

7. S. Alioli, C.W. Bauer, C.J. Berggren, A. Hornig, F.J. Tackmann, C.K. Vermilion et al., Combining higher-order resummation with multiple NLO calculations and parton showers in GENEVA. JHEP 09, 120 (2013). https://doi.org/10.1007/JHEP09(2013)120. arXiv: 1211.7049

8. L. Lönnblad, S. Prestel, Merging multi-leg NLO matrix elements with parton showers. JHEP 03, 166 (2013). https://doi.org/10. 1007/JHEP03(2013)166. arXiv:1211.7278

9. J. Alwall, R. Frederix, S. Frixione, V. Hirschi, F. Maltoni, O. Mattelaer et al., The automated computation of tree-level and nextto-leading order differential cross sections, and their matching to parton shower simulations. JHEP 07, 079 (2014). https://doi.org/ 10.1007/JHEP07(2014)079. arXiv:1405.0301

10. F. Cascioli, P. Maierhofer, S. Pozzorini, Scattering amplitudes with open loops. Phys. Rev. Lett. 108, 111601 (2012). https://doi.org/ 10.1103/PhysRevLett.108.111601. arXiv:1111.5206

11. G. Cullen, N. Greiner, G. Heinrich, G. Luisoni, P. Mastrolia, G. Ossola et al., Automated one-loop calculations with GoSam. Eur. Phys. J. C 72, 1889 (2012). https://doi.org/10.1140/epjc/ s10052-012-1889-1. arXiv:1111.2034

12. S. Actis, A. Denner, L. Hofer, J.-N. Lang, A. Scharf, S. Uccirati, RECOLA: REcursive Computation of One-Loop Amplitudes. Comput. Phys. Commun. 214, 140 (2017). https://doi.org/10.1016/ j.cpc.2017.01.004. arXiv:1605.01090

13. C. Berger, Z. Bern, L. Dixon, F. Febres Cordero, D. Forde, H. Ita et al., An automated implementation of on-shell methods for oneloop amplitudes. Phys. Rev. D78, 036003 (2008). https://doi.org/ 10.1103/PhysRevD.78.036003. arXiv:0803.4180

14. S. Alioli, P. Nason, C. Oleari, E. Re, A general framework for implementing NLO calculations in shower Monte Carlo programs: the POWHEG BOX. JHEP 06, 043 (2010). https://doi.org/10.1007/ JHEP06(2010)043. arXiv:1002.2581

15. T. Gleisberg, S. Hoeche, F. Krauss, A. Schalicke, S. Schumann, J.-C. Winter, SHERPA 1. alpha: a proof of concept version. JHEP 02, 056 (2004). https://doi.org/10.1088/1126-6708/2004/02/056. arXiv:hep-ph/0311263

16. M. Bahr et al., Herwig++ Physics and Manual. Eur. Phys. J. C 58, 639 (2008). https://doi.org/10.1140/epjc/s10052-008-0798-9. arXiv:0803.0883

17. S. Alioli, C.W. Bauer, C. Berggren, F.J. Tackmann, J.R. Walsh, Drell-Yan production at NNLL'+NNLO matched to parton showers. Phys. Rev. D 92, 094020 (2015). https://doi.org/10.1103/ PhysRevD.92.094020. arXiv:1508.01475

18. C. Degrande, C. Duhr, B. Fuks, D. Grellscheid, O. Mattelaer, T. Reiter, UFO - the universal FeynRules output. Comput. Phys. Commun. 183, 1201 (2012). https://doi.org/10.1016/j.cpc.2012. 01.022. arXiv: 1108.2040

19. N.D. Christensen, C. Duhr, FeynRules-Feynman rules made easy. Comput. Phys. Commun. 180, 1614 (2009). https://doi.org/10. 1016/j.cpc.2009.02.018. arXiv:0806.4194

20. A. Alloul, N.D. Christensen, C. Degrande, C. Duhr, B. Fuks, FeynRules 2.0 - a complete toolbox for tree-level phenomenol- 
ogy. Comput. Phys. Commun. 185, 2250 (2014). https://doi.org/ 10.1016/j.cpc.2014.04.012. arXiv:1310.1921

21. C. Degrande, Automatic evaluation of UV and R2 terms for beyond the Standard Model Lagrangians: a proof-of-principle. Comput. Phys. Commun. 197, 239 (2015). https://doi.org/10.1016/j.cpc. 2015.08.015. arXiv: 1406.3030

22. S. Frixione, B. Fuks, V. Hirschi, K. Mawatari, H.-S. Shao, P. Sunder et al., Automated simulations beyond the Standard Model: supersymmetry. JHEP 12, 008 (2019). https://doi.org/10.1007/ JHEP12(2019)008. arXiv:1907.04898

23. R. Frederix, S. Frixione, S. Prestel, P. Torrielli, On the reduction of negative weights in MC@NLO-type matching procedures. arXiv:2002.12716

24. K. Hamilton, P. Nason, G. Zanderighi, MINLO: multi-scale improved NLO. JHEP 10, 155 (2012). https://doi.org/10.1007/ JHEP10(2012)155. arXiv:1206.3572

25. K. Hamilton, P. Nason, C. Oleari, G. Zanderighi, Merging H/W/Z +0 and 1 jet at NLO with no merging scale: a path to parton shower + NNLO matching. JHEP 05, 082 (2013). https://doi.org/10.1007/ JHEP05(2013)082. arXiv:1212.4504

26. K. Hamilton, P. Nason, E. Re, G. Zanderighi, NNLOPS simulation of Higgs boson production. JHEP 10, 222 (2013). https://doi.org/ 10.1007/JHEP10(2013)222. arXiv:1309.0017

27. P.F. Monni, P. Nason, E. Re, M. Wiesemann, G. Zanderighi, $M_{1 N N L O}{ }_{P S}$ : a new method to match NNLO QCD to parton showers. JHEP 05, 143 (2020). https://doi.org/10.1007/ JHEP05(2020)143. arXiv:1908.06987

28. P.F. Monni, E. Re, M. Wiesemann, MiNNLOPs: Optimizing $2 \rightarrow 1$ hadronic processes. arXiv:2006.04133

29. R. Frederix, S. Frixione, Merging meets matching in MC@NLO. JHEP 12, 061 (2012). https://doi.org/10.1007/JHEP12(2012)061. arXiv: 1209.6215

30. S. Alioli, C.W. Bauer, C. Berggren, F.J. Tackmann, J.R. Walsh, S. Zuberi, Matching fully differential NNLO calculations and parton showers. JHEP 06, 089 (2014). https://doi.org/10.1007/ JHEP06(2014)089. arXiv:1311.0286

31. S. Höche, Y. Li, S. Prestel, Drell-Yan lepton pair production at NNLO QCD with parton showers. Phys. Rev. D 91, 074015 (2015). https://doi.org/10.1103/PhysRevD.91.074015. arXiv:1405.3607

32. T. Ježo, P. Nason, On the treatment of resonances in nextto-leading order calculations matched to a parton shower. JHEP 12, 065 (2015). https://doi.org/10.1007/JHEP12(2015)065. arXiv: 1509.09071

33. R. Frederix, S. Frixione, A.S. Papanastasiou, S. Prestel, P. Torrielli, Off-shell single-top production at NLO matched to parton showers. JHEP 06, 027 (2016). https://doi.org/10.1007/JHEP06(2016)027. arXiv: 1603.01178

34. J.M. Campbell, R. Ellis, R. Frederix, P. Nason, C. Oleari, C. Williams, NLO Higgs boson production plus one and two jets using the POWHEG BOX, MadGraph4 and MCFM. JHEP 07, 092 (2012). https://doi.org/10.1007/JHEP07(2012)092. arXiv: 1202.5475

35. G. Luisoni, P. Nason, C. Oleari, F. Tramontano, $H W^{ \pm} / \mathrm{HZ}+0$ and 1 jet at NLO with the POWHEG BOX interfaced to GoSam and their merging within MiNLO. JHEP 10, 083 (2013). https://doi. org/10.1007/JHEP10(2013)083. arXiv:1306.2542

36. G. Luisoni, C. Oleari, F. Tramontano, $W b \bar{b} j$ production at NLO with POWHEG+MiNLO. JHEP 04, 161 (2015). https://doi.org/10. 1007/JHEP04(2015)161. arXiv:1502.01213

37. T. Ježo, J.M. Lindert, P. Nason, C. Oleari, S. Pozzorini, An NLO+PS generator for $t \bar{t}$ and $W t$ production and decay including non-resonant and interference effects. Eur. Phys. J. C 76, 691 (2016). https://doi.org/10.1140/epjc/s10052-016-4538-2. arXiv:1607.04538

38. R. Frederix, S. Frixione, V. Hirschi, D. Pagani, H.-S. Shao, M.Zaro, The automation of next-to-leading order electroweak calculations.
JHEP 07, 185 (2018). https://doi.org/10.1007/JHEP07(2018)185. arXiv:1804.10017

39. V. Hirschi, R. Frederix, S. Frixione, M.V. Garzelli, F. Maltoni, R. Pittau, Automation of one-loop QCD corrections. JHEP 05, 044 (2011). https://doi.org/10.1007/JHEP05(2011)044. arXiv: 1103.0621

40. G. Ossola, C.G. Papadopoulos, R. Pittau, Reducing full one-loop amplitudes to scalar integrals at the integrand level. Nucl. Phys. B 763, 147 (2007). https://doi.org/10.1016/j.nuclphysb.2006.11.012. arXiv:hep-ph/0609007

41. P. Mastrolia, E. Mirabella, T. Peraro, Integrand reduction of one-loop scattering amplitudes through Laurent series expansion. JHEP 06, 095 (2012). https://doi.org/10.1007/JHEP11(2012)128. arXiv: 1203.0291

42. G. Passarino, M. Veltman, One loop corrections for $e^{+} e^{-}$annihilation into $\mu^{+} \mu^{-}$in the Weinberg model. Nucl. Phys. B 160, 151 (1979). https://doi.org/10.1016/0550-3213(79)90234-7

43. A.I. Davydychev, A simple formula for reducing Feynman diagrams to scalar integrals. Phys. Lett. B 263, 107 (1991). https:// doi.org/10.1016/0370-2693(91)91715-8

44. A. Denner, S. Dittmaier, Reduction schemes for one-loop tensor integrals. Nucl. Phys. B 734, 62 (2006). https://doi.org/10.1016/j. nuclphysb.2005.11.007. arXiv:hep-ph/0509141

45. G. Ossola, C.G. Papadopoulos, R. Pittau, CutTools: a program implementing the OPP reduction method to compute oneloop amplitudes. JHEP 03, 042 (2008). https://doi.org/10.1088/ 1126-6708/2008/03/042. arXiv:0711.3596

46. T. Peraro, Ninja: automated integrand reduction via Laurent expansion for one-loop amplitudes. Comput. Phys. Commun. 185, 2771 (2014). https://doi.org/10.1016/j.cpc.2014.06.017. arXiv: 1403.1229

47. V. Hirschi, T. Peraro, Tensor integrand reduction via Laurent expansion. JHEP 06, 060 (2016). https://doi.org/10.1007/ JHEP06(2016)060. arXiv:1604.01363

48. A. Denner, S. Dittmaier, L. Hofer, Collier: a fortran-based Complex One-Loop LIbrary in Extended Regularizations. Comput. Phys. Commun. 212, 220 (2017). https://doi.org/10.1016/j.cpc.2016.10. 013. arXiv: 1604.06792

49. P. de Aquino, W. Link, F. Maltoni, O. Mattelaer, T. Stelzer, ALOHA: automatic libraries of helicity amplitudes for Feynman diagram computations. Comput. Phys. Commun. 183, 2254 (2012). https://doi.org/10.1016/j.cpc.2012.05.004. arXiv:1108.2041

50. S. Alioli, P. Nason, C. Oleari, E. Re, NLO Higgs boson production via gluon fusion matched with shower in POWHEG. JHEP 04, 002 (2009). https://doi.org/10.1088/1126-6708/2009/04/002. arXiv:0812.0578

51. F. Demartin, F. Maltoni, K. Mawatari, B. Page, M. Zaro, Higgs characterisation at NLO in QCD: CP properties of the top-quark Yukawa interaction. Eur. Phys. J. C 74, 3065 (2014). https://doi. org/10.1140/epjc/s10052-014-3065-2. arXiv:1407.5089

52. P. Artoisenet et al., A framework for Higgs characterisation. JHEP 11, 043 (2013). https://doi.org/10.1007/JHEP11(2013)043. arXiv: 1306.6464

53. F. Maltoni, K. Mawatari, M. Zaro, Higgs characterisation via vector-boson fusion and associated production: NLO and partonshower effects. Eur. Phys. J. C 74, 2710 (2014). https://doi.org/10. 1140/epjc/s10052-013-2710-5. arXiv:1311.1829

54. F. Demartin, F. Maltoni, K. Mawatari, M. Zaro, Higgs production in association with a single top quark at the LHC. Eur. Phys. J. C 75, 267 (2015). https://doi.org/10.1140/epjc/s10052-015-3475-9. arXiv:1504.00611

55. F. Demartin, B. Maier, F. Maltoni, K. Mawatari, M. Zaro, tWH associated production at the LHC. Eur. Phys. J. C 77, 34 (2017). https:// doi.org/10.1140/epjc/s10052-017-4601-7. arXiv:1607.05862 
56. R.D. Ball et al., Parton distributions with LHC data. Nucl. Phys. B 867, 244 (2013). https://doi.org/10.1016/j.nuclphysb.2012.10.003. arXiv: 1207.1303

57. M. Whalley, D. Bourilkov, R. Group, The Les Houches accord PDFs (LHAPDF) and LHAGLUE. In: HERA and the LHC: A Workshop on the Implications of HERA and LHC Physics (Startup Meeting, CERN, 26-27 March 2004; Midterm Meeting, CERN, 11-13 October 2004), pp. 575-581 (2005). arXiv:hep-ph/0508110

58. A. Buckley, J. Ferrando, S. Lloyd, K. Nordström, B. Page, M. Rüfenacht et al., LHAPDF6: parton density access in the LHC precision era. Eur. Phys. J. C 75, 132 (2015). https://doi.org/10. 1140/epjc/s10052-015-3318-8. arXiv:1412.7420

59. M. Cacciari, G.P. Salam, G. Soyez, The anti- $k_{t}$ jet clustering algorithm. JHEP 04, 063 (2008). https://doi.org/10.1088/1126-6708/ 2008/04/063. arXiv:0802.1189

60. M. Cacciari, G.P. Salam, G. Soyez, FastJet User Manual. Eur. Phys. J. C 72, 1896 (2012). https://doi.org/10.1140/epjc/ s10052-012-1896-2. arXiv:1111.6097

61. K. Hagiwara, Q. Li, K. Mawatari, Jet angular correlation in vector-boson fusion processes at hadron colliders. JHEP 07, 101 (2009). https://doi.org/10.1088/1126-6708/2009/07/101. arXiv:0905.4314

62. V. Del Duca, W. Kilgore, C. Oleari, C. Schmidt, D. Zeppenfeld, Gluon fusion contributions to $\mathrm{H}+2$ jet production. Nucl. Phys. B 616, 367 (2001). https://doi.org/10.1016/ S0550-3213(01)00446-1. arXiv:hep-ph/0108030

63. V. Del Duca, W. Kilgore, C. Oleari, C. Schmidt, D. Zeppenfeld, Higgs +2 jets via gluon fusion. Phys. Rev. Lett. 87, 122001 (2001). https://doi.org/10.1103/PhysRevLett.87.122001. arXiv:hep-ph/0105129

64. T. Plehn, D.L. Rainwater, D. Zeppenfeld, Determining the structure of Higgs couplings at the LHC. Phys. Rev. Lett. 88, 051801 (2002). https://doi.org/10.1103/PhysRevLett.88.051801. arXiv:hep-ph/0105325
65. G. Klamke, D. Zeppenfeld, Higgs plus two jet production via gluon fusion as a signal at the CERN LHC. JHEP 04, 052 (2007). https:// doi.org/10.1088/1126-6708/2007/04/052. arXiv:hep-ph/0703202

66. J.R. Andersen, K. Arnold, D. Zeppenfeld, Azimuthal angle correlations for Higgs boson plus multi-jet events. JHEP 06, 091 (2010). https://doi.org/10.1007/JHEP06(2010)091. arXiv:1001.3822

67. F. Campanario, M. Kubocz, D. Zeppenfeld, Gluon-fusion contributions to $\Phi+2$ jet production. Phys. Rev. D 84, 095025 (2011). https://doi.org/10.1103/PhysRevD.84.095025. arXiv:1011.3819

68. C. Englert, M. Spannowsky, M. Takeuchi, Measuring Higgs CP and couplings with hadronic event shapes. JHEP 06, 108 (2012). https://doi.org/10.1007/JHEP06(2012)108. arXiv:1203.5788

69. C. Englert, D. Goncalves-Netto, K. Mawatari, T. Plehn, Higgs quantum numbers in weak boson fusion. JHEP 01, 148 (2013). https://doi.org/10.1007/JHEP01(2013)148. arXiv:1212.0843

70. M.J. Dolan, P. Harris, M. Jankowiak, M. Spannowsky, Constraining $C P$-violating Higgs sectors at the LHC using gluon fusion. Phys. Rev. D 90, 073008 (2014). https://doi.org/10.1103/PhysRevD.90. 073008. arXiv: 1406.3322

71. V. Hankele, G. Klamke, D. Zeppenfeld, T. Figy, Anomalous Higgs boson couplings in vector boson fusion at the CERN LHC. Phys. Rev. D 74, 095001 (2006). https://doi.org/10.1103/PhysRevD.74. 095001. arXiv:hep-ph/0609075

72. A.V. Gritsan, J. Roskes, U. Sarica, M. Schulze, M. Xiao, Y. Zhou, New features in the JHU generator framework: constraining Higgs boson properties from on-shell and off-shell production. Phys. Rev. D 102(5), 056022 (2020). https://doi.org/10.1103/PhysRevD.102. 056022

73. R. Frederix, K. Hamilton, Extending the MINLO method. JHEP 05, 042 (2016). https://doi.org/10.1007/JHEP05(2016)042. arXiv: 1512.02663 